\title{
The Impact of the Preferential Attachment Level on the Innovation Network Structure and Innovation Efficiency
}

\author{
Lei Hua $\mathbb{D}^{1,2}$ Zhong Yang, ${ }^{2}$ Jiyou Shao, ${ }^{2}$ and Dan Jin ${ }^{2}$ \\ ${ }^{1}$ School of Management Science and Engineering, Nanjing University of Information Science and Technology, Nanjing 210044, \\ China \\ ${ }^{2}$ School of Business, Nanjing University, Nanjing 210093, China
}

Correspondence should be addressed to Lei Hua; hualei@nuist.edu.cn

Received 6 March 2021; Revised 19 July 2021; Accepted 16 September 2021; Published 24 September 2021

Academic Editor: Haoran Zhang

Copyright (C) 2021 Lei Hua et al. This is an open access article distributed under the Creative Commons Attribution License, which permits unrestricted use, distribution, and reproduction in any medium, provided the original work is properly cited.

\begin{abstract}
In the formation process of innovation networks, the level of preferential attachment between agents has an important impact on the resulting structural and functional characteristics of a network. A simulation model of innovation networks with variable preferential levels of attachment is established to study these impacts. Negative preferences tend to form chain-type structures, while positive preferences tend to form star-type structures. Furthermore, a simulation process of innovation is added to the network model to test the impact of the resulting structures on innovation efficiency. For explorative innovation, the structure with unbiased preference has the fastest innovation speed, but the structures with strong positive preference show lower innovation costs. For exploitive innovation, strong negative preferences generate the fastest innovation speed and relatively low cost, while strong positive preferences show a slightly lower cost than negative preference structures but a much lower innovation speed. Finally, some internal mechanisms of the results are discussed.
\end{abstract}

\section{Introduction}

Innovation networks can help individuals cooperate and obtain the sources required in the process of innovation and have thus become the main organizational form of modern innovation activities. As an essential element of innovations, innovation networks' structure will inevitably have an important impact on the innovation process. Social network theory has become an important tool for innovation research, and much comprehensive research on the structure of innovation networks has emerged. This kind of research can be divided into two perspectives: (1) how innovation networks are formed and (2) how the structure of the innovation network affects its function [1].

In the formation process of innovation networks, individuals select their partners with certain strategies, which eventually leads to different network structures. There are many reasons for individuals to choose their innovation partners, such as resource complementarity [2], social capital advantage [3], and competitive strategy [4]. For example, Fang [5] indicates that firms tend to give priority to those with large knowledge complementarity when choosing partners. Iturrioz et al. [6] indicate that individuals tend to preferentially choose partners with social capital advantages. Thus, in this process, preferential attachment becomes a key element to direct the growth of the network and its resulting structure.

The concept of preferential attachment in network science is closely related to another famous concept, namely, the Mathew effect, which describes the phenomenon that, in societies, the rich tend to become richer and the potent even more powerful. As the Mathew effect describes a general principle of various complex systems in nature and society, the relevant studies form a vibrant and long-standing research field. Many scholars observe the Mathew effect in different patterns of scientific collaboration [7], sociotechnical and biological networks [8-10], propagation of citations [8], emergence of scientific progress and impact [11], career longevity [12], and education and brain development [13]. 
Although the Mathew effect has been noticed for a very long time, its recently acquired prominence is largely due to the rise in the concept of preferential attachment in network science. Barabási and Albert [14] conducted a pioneering study on the important role of preferential attachment in network science. In their seminal work, they defined two important mechanisms of the formation process of complex networks: network growth and preferential attachment. In the famous Barabási-Albert (BA) model $[14,15]$, a new node joining a growing network can in principle connect to any preexisting node. However, preferential attachment dictates that its choice will not be entirely random but linearly biased by the number of links that the preexisting nodes have with other nodes. The most prominent feature of this model is that, for every node, the higher the degree is, the faster the number of its links increases. Thus, preferential attachment becomes a perfect interpretation of the Mathew effect in the context of network science and can even be treated equivalently in some studies of self-organization in biology and societies.

Detecting a power-law distribution or at least a related fat-tailed distribution in empirical data is consistent with the Mathew effect, and the probability distribution of a quantity $x$ that obeys a power law is $p(x) \sim 1 / x^{1+\mu}$ with $\mu>0$, where $\alpha=1+\mu$ is the scaling parameter. Measuring preferential attachment requires time-resolved data, and we need to be able to measure the rate at which the measured quantity $x$ (such as the number of links in the network context) increases. Assuming the change in $x$ over a short time interval $\Delta t$ is $\Delta x$, the mechanism of preferential attachment assumes that $\Delta x \sim A x^{\gamma}$, where $A$ is the attachment rate and $\gamma$ determines the nonlinearity of the attachment kernel $x^{\gamma}$. If $\gamma=1$, the attachment kernel is linear, and deviations of $\gamma$ below or above 1 yield sublinear and superlinear preferential attachment, respectively [14].

Based on this idea, scholars employ cumulation or averaging to remove the stochastic fingerprint of autocatalytic growth and to reliably estimate whether the process is governed by linear attachment [16]. Numerous studies with empirical data show that the value of $\gamma$ is quite different in empirical data. In scientific collaboration networks, data from different scientific databases of different disciplines reveal both superlinear and sublinear preferential attachments $[7,8,17,18]$. The network of movie actors in [8] shows growth characterized by $\gamma=0.81$. The sexual contact networks in [19] are governed by the growth of a sublinear preferential attachment with $0.5<\gamma<0.7$. The accumulation of citations to scientific papers reported in [20] is governed by superlinear preferential attachment with $1.25<\gamma<1.3$. The Worldwide Web has also been shown to display a pure power law over the entire web, but the distribution within sets of category-specific web pages is typically unimodal on a log scale, varying across different categories [21].

Different levels of preferential attachment result in different network structures. Sublinear preferential attachment gives rise to a stretched exponential cutoff, while superlinear attachment eventually results in a single entity of the system gaining complete monopoly [22, 23]. Different structures resulting from different levels of preferential attachment, on the other hand, will inevitably have an important impact on the function of the network. Numerous studies have tested the impact of corresponding network structures on knowledge transfer [24], innovation diffusion [25], epidemiology [26], economic outcomes [27], and so on.

These previous studies are of great significance for understanding the role of preferential attachment, but there are still some gaps that need to be filled. First, the impact of different levels of preferential attachment on network structure needs further exploration. The existing literature basically treats the preferential attachment and Mathew effect as similar concepts. The underlying assumption is that the new node is always more likely to be connected to the high-degree preexisting nodes. However, there are still some exceptions reported $[11,21,28]$, where the Mathew effect fails to dominate, but the mechanism is not adequately explained, especially in the sense of preferential attachment. What happens when new nodes are prone to connect to lowdegree preexisting nodes? Although this possibility is to a degree rooted in intuition and lacks empirical support at present, it is worth exploring to support the theory. Second, the impact of different levels of preferential attachment on the overall function of the network needs further exploration. The Mathew effect makes the rich tend to get richer and the potent even more powerful, but is this what people truly want? For example, the Mathew effect begets inequality in some social and economic systems, and some precautions should be taken by the government to mitigate this effect. However, there is still a lack of relevant research from this perspective on this topic.

In the context of innovation networks, especially, the question of what lines of networks are more conductive to innovations is a basic theoretical problem in this field. Many scholars have explored the impact of various structural characteristics of the innovation process, such as strong and weak ties [29, 30], structural holes [31, 32], cliquishness $[33,34]$, connectivity $[35,36]$, and network density $[37,38]$. However, the role of preferential attachment is still not fully explored.

Therefore, this paper explores the characteristics of the network structure under different levels of preferential attachment and further discusses the influence of the resulting structures on innovation efficiency. The specific approach is as follows: first, based on the classic scale-free network model, a network simulation model with variable network preference is constructed to analyze the network structure characteristics generated under different levels of preferential attachment. Second, a simulation model of the innovation process is established and run under different networks formed by different preferential attachment levels. Third, the influences of different preferences of attachments on network structure and innovation efficiency are discussed.

\section{The Impact of the Preferential Attachment Level on the Innovation Network Structure}

2.1. Network Model with Variable Levels of Preferential Attachment. Based on the classic BA model, the model here 
with variable levels of preferential attachment also includes the two-generation mechanisms of network growth and preferential attachment.

First, the growth mechanism here is the same as that of the BA model. Initially, a small number $\left(m_{0}\right)$ of nodes are generated and fully connected with each other. At each subsequent time $t$, a new node is added to the network, and $m$ existing nodes $\left(m<m_{0}\right)$ are selected as its new neighbors to create links. After $t$ steps, the network evolves into a structure with the total number of nodes as $N=m_{0}+t$ and the number of edges as $C_{m_{0}}^{2}+t m$.

Second, the preferential attachment mechanism is an expansion version of the BA model. In the BA model, at each time step, if the degree of existing node $i$ is $k_{i}$, the probability of node $i$ being selected as the neighbor of the new node is

$$
p\left(k_{i}\right)=\frac{k_{i}}{\sum_{j} k_{j}} .
$$

The network model with variable levels of preferential attachment here improves this setting by introducing a new parameter $\theta$ to adjust the level of preference rate, especially in the context of innovation networks of this study. Thus, the probability $p\left(k_{i}\right)$ above is transformed into

$$
\pi\left(k_{i}\right)=\frac{k_{i}^{\theta}}{\sum_{j} k_{j}^{\theta}} .
$$

Now, the probability of establishing a connection between an existing node and a new node is also determined by the preference level $\theta$. Theoretically, the range of $\theta$ is $(-\infty$, $+\infty$ ). When $\theta<0$, new nodes were more likely to choose the nodes with a lower degree to establish connections, and this tendency increased with the decrease in the $\theta$ value. When $\theta=0$, the probabilities of each existing node being selected as link neighbors by the new node are equal, which supports the random connection mechanism mentioned in the existing literature $[21,39]$. When $\theta=1,(2)$ is equivalent to (1), making the preference of attachment linearly proportional to the node degree. Finally, $\theta>1$ indicates a stronger trend than proportional, as in the superlinear preferential attachment mentioned previously.

\subsection{Network Structures Caused by Different Levels of Pref-} erential Attachment. We use NetLogo [40] software to program and run the model. For all the parameters in the model that need to be initialized, we first clarify their possible range through theoretical analysis and then decide the proper experimental values that could clearly reveal the relationships through repeated experiments. For example, the theoretical value range of the preference intensity $\theta$ is $(-\infty,+\infty)$. However, after a series of repeated experiments, it is found that, with other parameters fixed, the results are clear and worth discussion within the range of $(-7,2)$. Beyond that range, although the effect of $\theta$ remains, it becomes too nuanced to discriminate. Therefore, in the simulation process, when the value of the preference level is negative, we use the values of $\theta=-1,-3,-5,-7$. When the preference level is not negative, we use the values of $\theta=0,0.5,1,1.5,2$. The values of other parameters are set with the same method. The number of initial nodes in the network is $m_{0}=1$, and the number of edges formed by each new node is $m=1$. We run each network 500 time steps (including the initial step to generate the first starting node) to generate the same size of networks with different structures. The resulting network structures and corresponding degree distributions caused by different levels of preferential attachment are shown in Figures 1 and 2.

The simulation result shows that the level of preferential attachment has an important impact on the network structure.

First, from the perspective of the structural graph (Figure 1), when the preference intensity is negative and the value is small (e.g., $\theta=-7$ ), the network structure presents chain-type characteristics. When the preference level is positive and the value is large (e.g., $\theta=2$ ), the network shows star-type structural features. As the level of preference increases from small to large, hubs in the network emerge and grow and finally become a single superhub. In this process, the chain feature of the network structure gradually weakens, and the star feature gradually strengthens.

Second, from the perspective of the degree distribution of the networks (Figure 2), when the preference intensity is negative and the value is small (e.g., $\theta=-7$ ), the degree distribution reaches its peak at 2 , which means most nodes have a degree of 2, and a small number of nodes have a degree of 1 or 3 . When the preference level is positive and the value is large (e.g., $\theta=2$ ), the distribution reaches the peak at 1 , and the skewness of the distribution becomes very prominent. Most nodes are one-degree nodes, and nodes with remarkably high degrees exist. As the level of preference increases from small to large, the peak of the degree distribution gradually deviates from 2 to 1 , and the skewness becomes increasingly prominent.

Although the range of $\theta$ in the experiment is $[-7,2]$, it is easy to infer that if the preference level $\theta \longrightarrow-\infty$, each new node will be extremely inclined to choose low-degree nodes to create links and result in a single line structure of the network. As $\theta \longrightarrow+\infty$, each new node will be extremely inclined to create links with high-degree nodes and result in a perfect star structure. Therefore, if the number of simulation steps $t$ goes to infinity, which means that the total number of nodes $N$ goes to infinity, it is obvious that the degree distributions of the network are as follows.

When $\theta \longrightarrow-\infty$,

$$
\lim _{N \longrightarrow+\infty} p(k)= \begin{cases}1, & k=2, \\ 0, & k \neq 2 .\end{cases}
$$

When $\theta \longrightarrow+\infty$,

$$
\lim _{N \longrightarrow+\infty} p(k)= \begin{cases}1, & k=1, \\ 0, & k \neq 1 .\end{cases}
$$

According to the analysis in the previous section, when $\theta=1$, this model is equivalent to the classic BA model. 

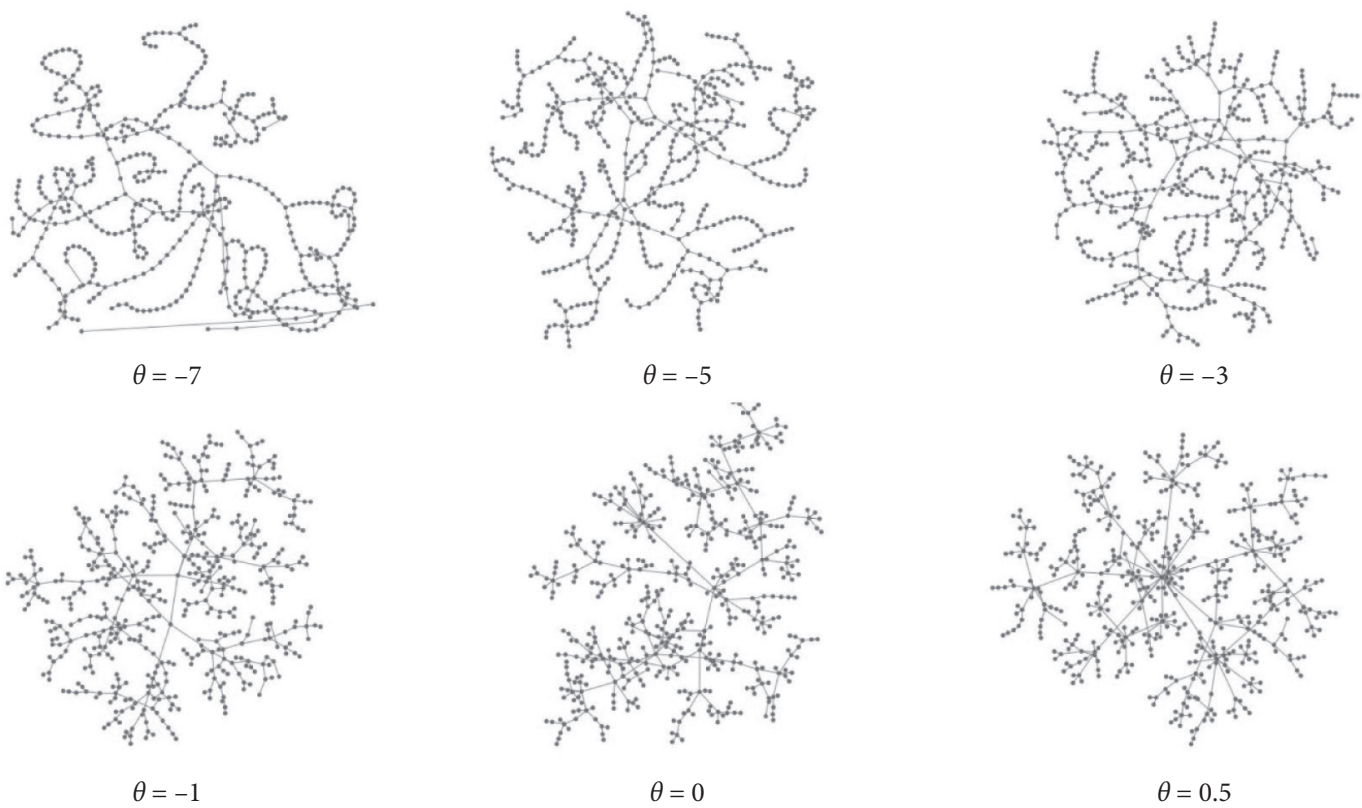

$\theta=0.5$
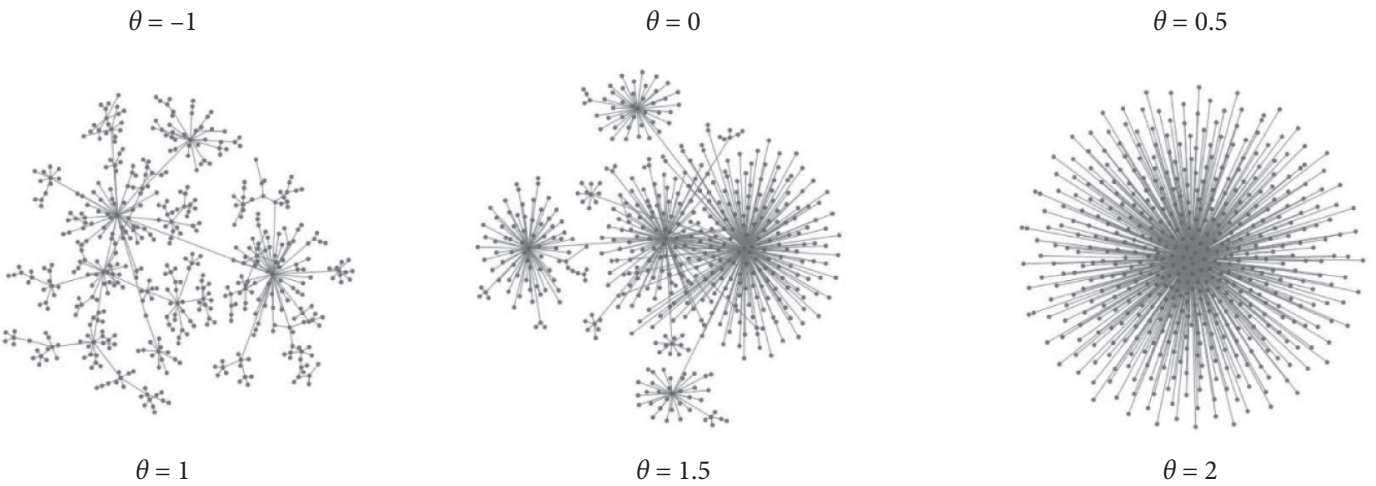

FIGURE 1: Illustration of network structures with different levels of preferential attachment. Each network is generated with corresponding values of parameter $\theta$ and the network size $N=500$.

\section{Innovation Efficiency of the Network Structures with Different Preferential Levels}

After elaborating the impact of preferential attachment on network structure, we explore its impact on innovation efficiency in the context of innovation networks. In this section, an innovation simulation model is established and run with the different structures generated above to observe the efficiencies of innovations.

\subsection{The Computational Representation of the Innovation} Process. Based on the consensus that innovation is a process of knowledge search, recombination, and selection, many scholars have established corresponding innovation models in different research contexts. Cowan's model [33] focuses on the diffusion of knowledge in the innovation process. In this model, the agents communicate and learn from each other under the constraints of network structure to acquire the knowledge needed for innovation. This process will constantly produce new ideas. However, as Fleming [41] pointed out, these new ideas are not always feasible, so there needs to be a selection mechanism to screen out those results that meet the requirements of the market. The model of Lovejoy and Sinha [42] focuses on describing the selection mechanism of new knowledge combination in the innovation process. Hua and Wang [34] combine these ideas and establish a model that includes the mechanisms of knowledge search, reorganization, and selection. In this paper, the model of the innovation simulation process is constructed with the idea of Hua and Wang's model. This model includes the following three steps.

First, the basic settings of knowledge and network structures are described.

Assume that all the knowledge needed to result in an innovation constitutes a specific knowledge vector $L=k_{1}, k_{2}, \ldots, k_{l}$. Each dimension of the vector represents a kind of knowledge needed to generate the innovation, and the values of $k_{1}$ to $k_{l}$ represent the amount of needed knowledge. Without losing generality, let $k_{1}=k_{2}=\cdots=k_{l}=k$.

Then, consider an undirected connected network with $N$ nodes, in which each node represents an innovation agent. Each agent $i$ has an $l$-dimensional knowledge vector $v_{i}=$ 

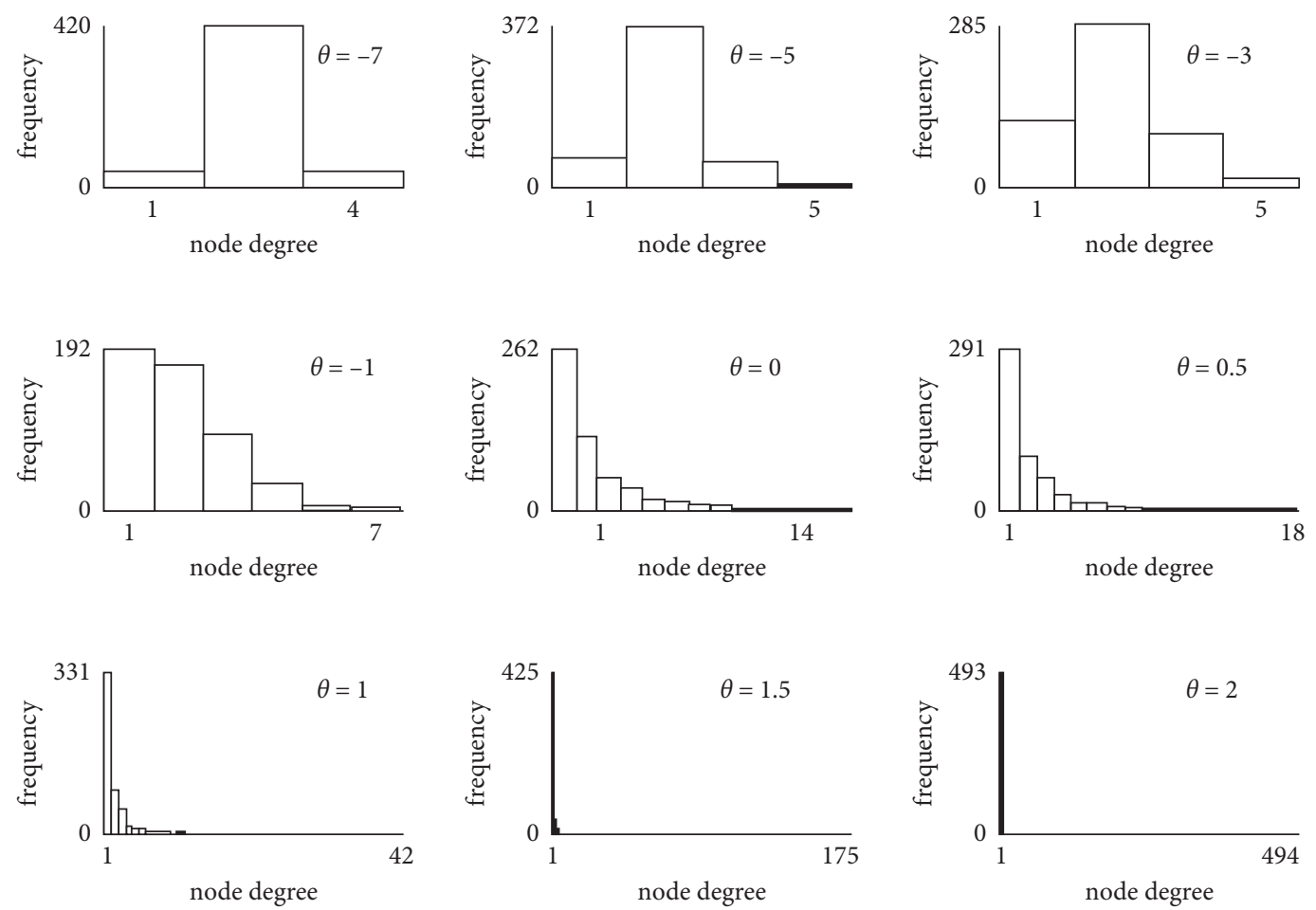

FIgURE 2: Degree distribution of network structures with different levels of preferential attachment. The nine distributions here correspond to the nine network structures in Figure 1.

$v_{i 1}, v_{i 2}, \ldots, v_{i 1}$ corresponding to $L$, the vector of innovation. Each component, $v_{i j}$, represents the knowledge quantity of dimension $j$ possessed by agent $i$. In the initial state, $l$ nodes are randomly selected from the population of $N$, and their knowledge vectors are set as follows. Randomly select a dimension $l$ from the knowledge vectors of each node, set the knowledge quantity of this dimension to a specific value $k_{j}$, set the other dimensions to 0 , and guarantee the linear independence of $l$ knowledge vectors. At the same time, set the knowledge vectors of the other $N-l$ nodes as 0 vectors. The meaning of this setting is that, in the initial state, the knowledge needed for innovation is scattered among different agents all over the network.

Second, the dynamics of the simulation process are shown.

At each time $t$, every agent randomly selects one of its link neighbors and communicates with it. When nodes $i$ and $j$ communicate with each other, they randomly select one dimension $c$ among all $l$ dimensions of the knowledge vector. If node $j$ has more knowledge in this dimension than node $i\left(v_{i c}<v_{j c}\right)$, then node $i$ will learn from node $j$ in this conversation, giving $v_{i c}$ an increment $\mathrm{d} v$. Similarly, $v_{j c}$ will get an increment $\mathrm{d} v$ if $v_{j c}<v_{i c}$. It is assumed that each node can communicate only on one topic each time due to the limit of communication ability. When there are no idle nodes in the network that can communicate with each other, time $t$ ends. In this process, knowledge flows over different agents, diffuses, and reorganizes with the constraints of network structure.

Third, the innovation process is terminated, and innovation efficiency is measured.
Innovation occurs when the knowledge vector of one node in the network becomes $L$. At this point, $v_{i}$ equals $L$, which means that the knowledge vector of this node is consistent with the knowledge vector needed by the innovation. This setting realizes the selection mechanism of knowledge combinations in the innovation process, which means that only the knowledge combination that meets the requirements of the innovation truly counts.

Speed and cost are both important measures of the efficiency of innovations. We use the method of Lovejoy and Sinha [42] to measure them. Let TTF (time to finish) denote the number of simulation steps from the initial state to the time that innovation occurs. Then, TTF measures the time needed to generate innovation and reflects the speed of innovation. Let NCTF (number of conversations to finish) denote the total conversations between agents throughout the whole innovation process. Then, NCTF measures the cost of innovation. Effective innovation networks are those with lower time and cost.

The knowledge dynamics of the innovation process described by this model are shown in Figure 3.

This innovation model has the following important characteristics.

First, the topics are randomly chosen while the agents communicate with each other. The implicit assumption is that innovations are somewhat unpredictable before they happen. People do not know in advance what combination of knowledge is required. This setting reflects the novelty of the required knowledge combination.

Second, in this model, innovation occurs when the vector of one agent meets the requirement of innovation. 


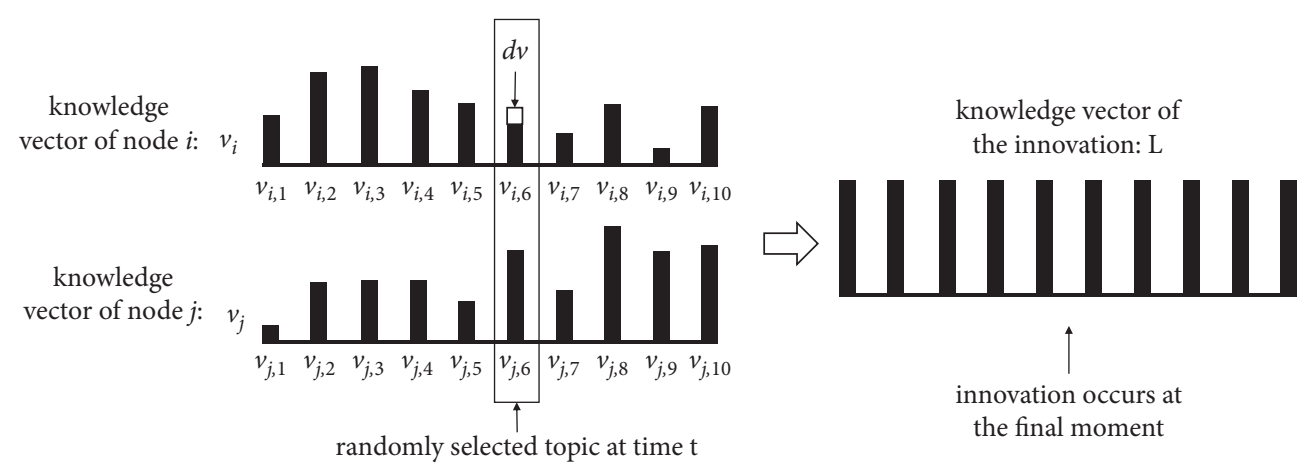

Figure 3: The knowledge dynamics of the innovation process. The left-hand side depicts the conversation between two nodes at one time step. Over time, once the knowledge vector of one node becomes equal to the innovation knowledge vector on the right-hand side, innovation occurs, and the innovation process terminates.

At this point, the simulation stops, rather than waiting until all the knowledge of the agents meets the requirement. Once innovation occurs, its knowledge dynamics will fundamentally change. On the one hand, when an agent produces innovation, it will adopt some leading strategies to protect and enjoy the benefits of innovation exclusively, such as applying for patents, which will inhibit other agents from producing the same innovation. On the other hand, when an innovation opportunity is found, it will lose its novelty, and other agents can quickly acquire relevant knowledge through imitation or purposeful learning. This model focuses on the generation process of innovation to avoid more discussion on the dynamics of knowledge after innovation.

3.2. Two Different Kinds of Innovations. The setting of the knowledge vector $L$ required for the innovation enables this model to distinguish the two different kinds of innovations, which are explorative innovation and exploitative innovation. Exploration and exploitation are a pair of important concepts in the research fields of organizational learning [43], innovation [44], and entrepreneurship [45]. March [46] proposed the concepts of explorative learning and exploitative learning in the study of organizational learning. $\mathrm{He}$ and Wong [47] defined the concepts of explorative innovation and exploitative innovation based on the analytical framework of March.

Explorative innovation emphasizes acquiring new knowledge and strives to go beyond the existing knowledge base [43]. Explorative innovation is realized mainly through expanding the width of knowledge rather than the depth of knowledge. Explorative innovation needs a variety of different types of knowledge but not the mass of each specific kind of knowledge.

Exploitative innovation is based on the existing knowledge and emphasizes the extraction, integration, strengthening, and improvement of the existing knowledge [43]. Exploitative innovation is realized mainly through increasing the depth of knowledge rather than the width of knowledge. Exploitative innovation consists of the accumulation of knowledge in some specific areas but not the diversity of knowledge.
In this model, the two important parameters, $l$ and $k$, in the vector of innovation $L$ represent the width and depth of knowledge, respectively. When the value of $l$ is relatively large and the value of $k$ is relatively small, it indicates that there are many types of knowledge needed to generate innovation, but the requirement for the knowledge quantity of each kind is low. Therefore, this setting represents explorative innovation. When the value of $l$ is relatively small and the value of $k$ is relatively large, the diversity of knowledge required for innovation is relatively small, and the quantity of specific types of knowledge is high. Therefore, this setting represents exploitative innovation. Figure 4 shows the difference in the knowledge vectors of the two different kinds of innovations.

3.3. Parameter Settings and Simulation Results. By running the above innovation model on different network structures, the innovation efficiency under different network structures can be observed.

In the network model, there are four exogenous variables that need to be set, namely, network size $N$, initial number of nodes $m_{0}$, number of new connections $m$, and attachment preference intensity $\theta$. Here, adopting the principle of the parameter settings mentioned above, let $N=500, m_{0}=1$, $m=1$, and $\theta \in[-10,10]$.

In the innovation model, the parameters $l$ and $k$ of innovation vector $L$ and the node knowledge increment $\mathrm{d} v$ in the communication process need to be initialized. For explorative innovation, the number of kinds of knowledge (i.e., knowledge kinds) is relatively large, while the quantity of each kind of knowledge is relatively low. Therefore, the number of knowledge kinds is set as $l=100$, and the number of knowledge quantities is $k=1$. For exploitative innovation, due to the relatively few kinds of knowledge and high knowledge quantity required, the number of knowledge kinds is set as $l=2$, and the knowledge quantity required for innovation is $k=100$. In addition, let the increment of knowledge in each conversation between nodes $\mathrm{d} v=1$.

Here, we also use the simulation software NetLogo [40] to program, and calibration runs suggest that we could replicate each experiment 100 times to generate sufficient margins of error to support statistical testing. 


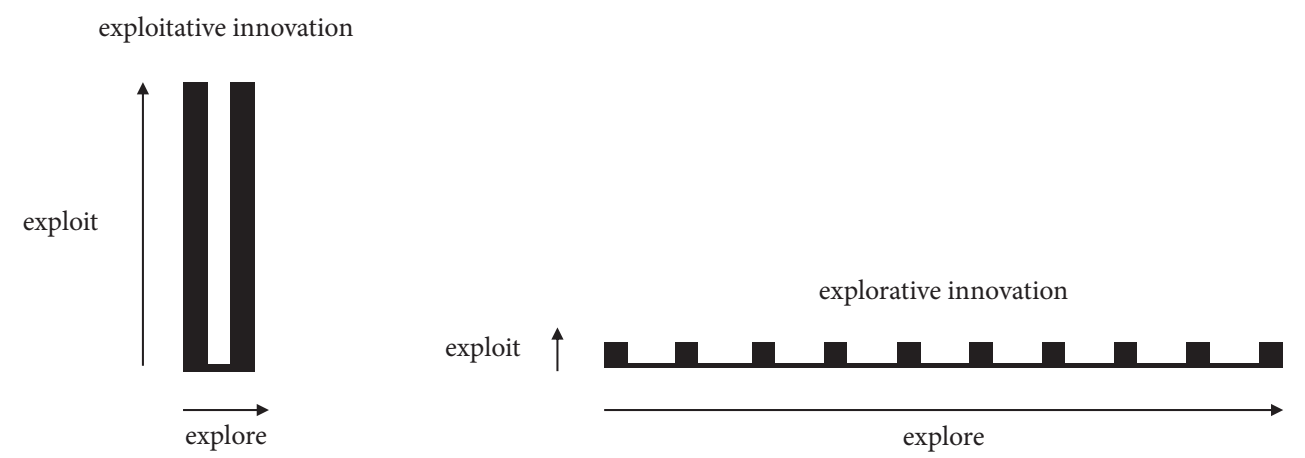

FIGURE 4: Illustration of the difference in knowledge vectors of the two different kinds of innovations. There are two acting regimes taking effect in the process of innovations: exploration, which broadens knowledge, and exploitation, which deepens knowledge.

Figures 5 and 6 show the TTF and NCTF of explorative innovation as a function of preference level $\theta$, which controls the structure of the network. The curve in the figure shows the mean value of TTF or NCTF, and the small vertical line segment represents the standard deviation at each point on the curve.

As seen from Figure 5, when the preference level $\theta<0$, the TTF of explorative innovation decreases with the increase of $\theta$. When the preference level $\theta>0$, the TTF of explorative innovation first increases rapidly with the increase in $\theta$ and then turns into a stable state at a higher level. When $\theta=0$, TTF reaches the minimum value. This result shows that, for explorative innovation, the network formed with no preference is the fastest structure to generate explorative innovations. Both positive and negative levels of preference will slow down the speed of innovation.

As seen from Figure 6, when the preference level $\theta<-1$, the NCTF of explorative innovation decreases with the increase in $\theta$. When the preference intensity $-1<\theta<3$, the NCTF of explorative innovation shows an upward pulse that peaks at $\theta=1$. When the preference intensity $\theta>3$, the NCTF dropped to a lower level than that before the pulse and continued to decline slowly as $\theta$ increased. It is worth mentioning that the peak of the pulse in Figure 6 happens to be the case of the BA network. This result shows that, for explorative innovation, a strong positive preference for attachment is more conducive to reducing innovation costs. However, when the preference approaches $\theta=1$, which means that the structure approaches linear preferential attachment, the innovation costs increase sharply. Therefore, the BA network is apparently not an ideal structure for explorative innovations.

Figures 7 and 8 show the TTF and NCTF of exploitative innovation as a function of parameter $\theta$.

As shown in Figure 7, when the preference level $\theta<0$, the value of TTF increases with the increase in $\theta$. When the preference level $\theta>0$, the TTF of exploitative innovation first increases rapidly with the increase in $\theta$ and then continues to increase slowly at a higher level. This result shows that, for exploitative innovation, negative values of preference level are more conducive to high innovation speed than positive values of $\theta$. When the preference level is

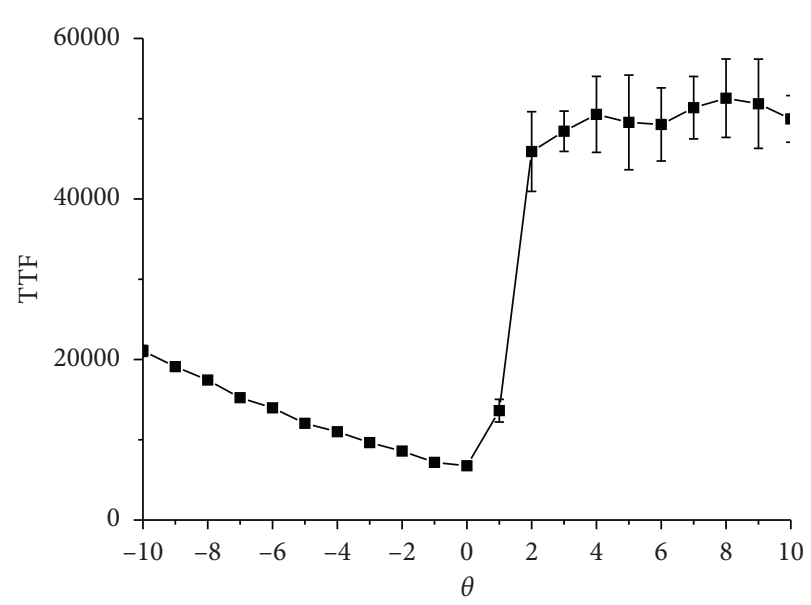

Figure 5: The measure of innovation speed TTF of explorative innovation as a function of the level of preferential attachment $\theta$.

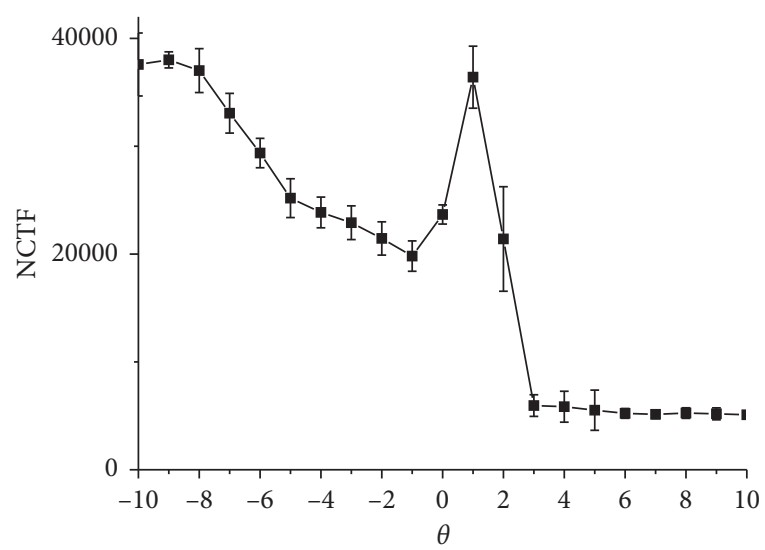

Figure 6: The measure of innovation cost NCTF of explorative innovation as a function of the level of preferential attachment $\theta$.

negative, the greater the intensity of preference (the absolute value of $\theta$ ), the faster the speed of innovation.

As shown in Figure 8, when the preference level $\theta<1$, the NCTF of exploitative innovation increases with the increase in $\theta$. When the preference level $\theta>1$, the NCTF of 


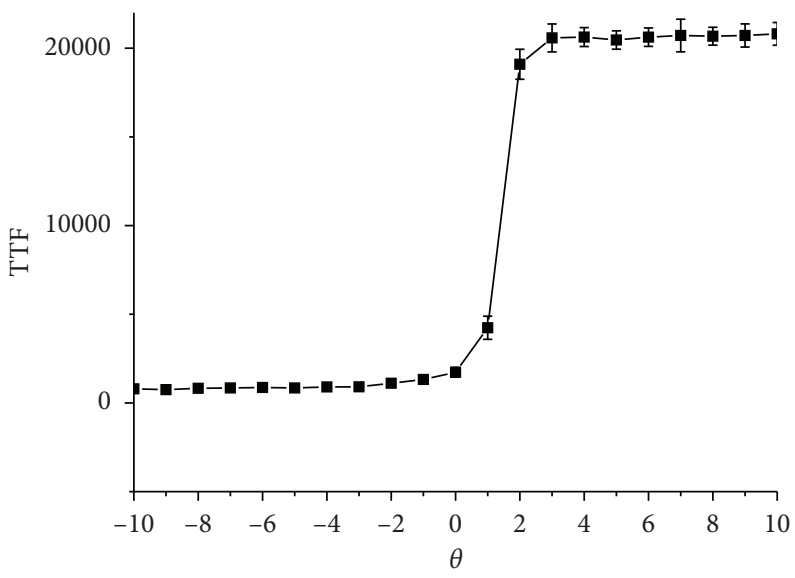

FIgURE 7: The measure of innovation speed TTF of exploitative innovation as a function of the level of preferential attachment $\theta$.

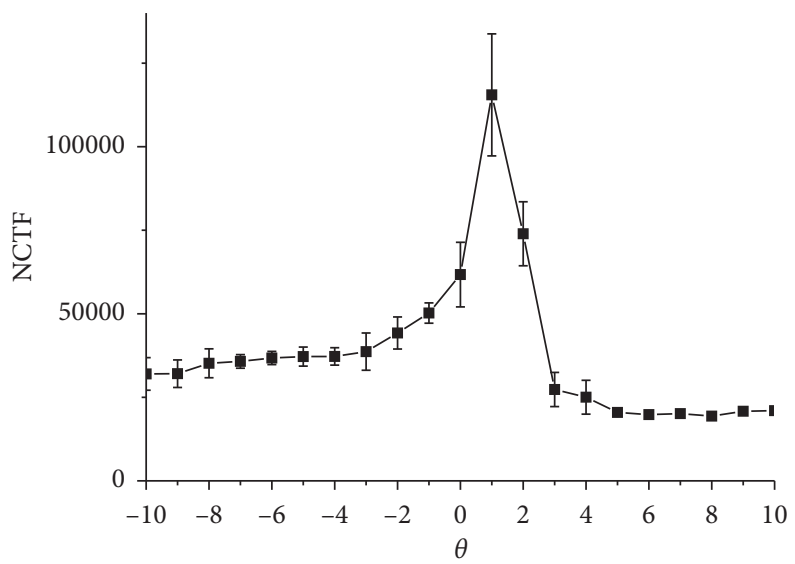

FIgURE 8: The measure of innovation cost NCTF of exploitative innovation as a function of the level of preferential attachment $\theta$.

exploitative innovation decreases with the increase in $\theta$. Regardless of whether the value of the preference level is positive or negative, if the preference intensity (absolute value of $\theta$ ) increases, the innovation cost will decrease. Large positive preferences are slightly better than their negative counterparts.

3.4. Further Discussion of Simulation Results. We explain the mechanism that leads to these simulation results from two perspectives: (1) the influence of network preference on the network structure and (2) the influence of the corresponding network structure on the knowledge dynamics in the innovation process.

The perspective of the influence of preference level $\theta$ on network structure is considered. As $\theta$ increases, nodes with large degrees start to appear and turn into hubs and even turn out to be a single superhub with a large positive value of $\theta$. This is consistent with the aforementioned literature that confirms the equivalence between preferential attachment and the Mathew effect. However, to explain the structural and functional mechanisms in the context of innovation networks, we need to turn to a deeper interpretation of this with some more concrete concepts in network science. Different levels of preferential attachment mainly affect three aspects of network structure: the shortest path length [33], network bottleneck [48], and conversational churning [42] between agents. The differences, created by different forms of preferential attachment, can be spotted at a glance in the structure of the resulting networks, as shown in Figure 1. The smaller the value of $\theta$, the more obvious the chain-type structure of the network. Then, the shortest path length between any two points on the network is longer. The larger the value of $\theta$, the more obvious the star-type structure of the network. Therefore, the central nodes form greater bottlenecks of the network, which inhibit the flow of knowledge. At the same time, the central nodes are connected to more peripheral nodes as $\theta$ increases, thus leading to more conversational churning of the central nodes, which means changing communication partners frequently.

The perspective of network structure's influence on knowledge dynamics is considered. First, the shorter the length of the characteristic path on the network is, the more conducive it is to improve the innovation speed and reduce the innovation cost $[33,34]$. At this time, nodes on the network can more easily acquire knowledge from other 
nodes. Second, network bottlenecks will hinder the flow of knowledge on the network. As pointed out by Gibson [48], high node centrality will lead to diffusion bottlenecks because peripheral nodes can only wait for the attention of central nodes due to fewer conversation partners. This will make the central node become the bottleneck of information transmission, thus reducing the speed of knowledge flow. However, network bottlenecks also have a positive side. Bottlenecks reduce communication opportunities between peripheral nodes so that most communications take place between peripheral nodes and central nodes, which greatly reduces redundant communication on the network and thus saves innovation costs. Third, the influence of conversational churning on the innovation process varies according to the types of innovation. For explorative innovation, nodes are required to expand the knowledge width as much as possible, but the requirements of specific fields are not remarkably high, so conversational churning is beneficial to the innovation process. For exploitative innovation, it is necessary to minimize conversational churning. Because innovators are required to dig deep into specific knowledge fields at this time, they need to communicate frequently with a small number of fixed partners.

In addition, it can be seen from the relationship between $\theta$ and the network structure that when $\theta<0$, the characteristic path length and conversational churning of the network change rapidly with $\theta$, while the change in bottleneck is not as obvious. When $\theta>0$, the bottleneck and conversational churning change obviously, while the shortest path length is already short, and the change is no longer obvious. The above reasons together lead to the simulation results shown in Figures 5-8.

\section{Robust Test}

The robustness of the results is discussed in the following two aspects.

First, other values of all exogenous variables involved in the model are tested. We rerun the above model with network size $N=200,400,600,800$, starting node number of networks $m_{0}=1,2,3$, and growth rate of network size $m=1,2,3$. The experimental results show that our conclusion is robust at least at a moderate level of variation around the parameter values of the basic model. For knowledge kinds $l$ and knowledge quantity $k$, their ratio determines the type of innovation. We run the tests of these two parameters under different ratios. The results also show that the conclusions are robust in the vicinity of the basic model with moderate levels of parameter change.

Second, other mechanisms of knowledge flow in the communication process of agents are tested. In the simulation process, when knowledge flows among agents, the quantity of knowledge acquired during learning from other agents each time is set as $\mathrm{d} v=1$, which is consistent with the setting of Lovejoy and Sinha [42] in their knowledge flow model. In addition, we also test the situation in the Cowan and Jonard [33] model, in which $\mathrm{d} v$ changes with the knowledge level of the learner or the situation of $\mathrm{d} v$ is a certain proportion of the difference in agents' knowledge. All these modifications result in conclusions with the same nature as the basic model, so they all pass the robustness test.

\section{Discussions and Conclusions}

This study is devoted to better understanding innovation network structure by means of preferential attachment, in particular the different levels of the attachment rate. The study consists of two parts that are intricately connected. The first part is what kind of network structures will be formed under different levels of preferential attachment. The second part is which of these structures is more conducive to generating innovations.

In the first part, based on the classic BA model, this study constructs a network model with variable preference of attachments, which can generate network structures with different levels of preferential attachment, and the corresponding structural characteristics of these networks are analyzed. It is found that the level of attachment preference will have an important influence on the network structure. High negative attachment preference tends to form chaintype structures. High positive attachment preference tends to form star-type structures. When the attachment preference changes from negative to positive and from small to large, the chain features of the network structure gradually weaken, and the star feature gradually strengthens.

In the second part, this paper constructs a simulation model of the innovation process and runs it on different network structures to compare the innovation efficiency of different network structures formed by different preferences of attachment. It is found that, for explorative innovation, a structure with zero preference can generate the fastest innovation speed, but a strong positive preference can generate the lowest innovation cost. For exploitative innovation, a strong negative attachment preference can produce the fastest innovation speed, and the innovation cost is also relatively low. However, although the innovation speed of strong positive preference is slow, its innovation cost can be slightly lower than that of strong negative preference.

The reasons for the above results are also analyzed. First, from the perspective of the influence of the preference level on the network structure, three key structural characteristics that affect innovation efficiency, namely, the shortest path length, network bottleneck, and conversational churning, are discussed. Then, the influence of these three structural characteristics on the knowledge dynamics is analyzed, and the internal mechanism of why these different network structures show different innovation performances is explained.

The main theoretical contribution of this paper is to introduce the variable preference of attachment into the innovation network model. On the one hand, this study explores how different levels of preferential attachment influence the network structures, especially the negative values of preferential levels. As the concept of preferential attachment is treated as a synonym for the Mathew effect in most existing literature, this study expands the meaning of "preference" and explores the effect in a wider theoretical boundary. Although most existing empirical studies observe 
positive preferences, some exceptions have been reported, which potentially reflects the necessity of this idea adopted in this study. Therefore, this model can help multidisciplinary researchers explore situations in which the Mathew effect is either applicable or not. On the other hand, this study explores how different levels of preferential attachment influence the function of the whole system, specifically, the innovation efficiency of innovation networks. Regarding the "effect of the Mathew effect," the extant literature mainly focuses on the welfare of individuals, such as the rich-getricher effect or the discrepancies benefiting individuals. However, few studies have explored the impact of preferential attachment on other important functions of the entire system. This study focuses on the field of innovation networks, and the conclusion also contributes to innovation theory. The results of this research shed light on the question of how innovators should choose their partners while pursuing different types of innovations. This idea can also be applied to the study of preferential attachment in other disciplines.

The practical significance of this paper mainly lies in the field of innovation strategy. The relations between preferential attachment and two acting regimes taking effect in the process of innovation, namely, exploration and exploitation, are explored. The conclusions have a certain enlightened significance for innovation policy makers if they could influence the preference of attachments with some policy instruments. Organizations pursuing explorative innovation need to make a choice between innovation speed and innovation cost. The attachment strategy without preference produces the fastest innovation speed, while the attachment strategy with strong positive preference produces the lowest innovation cost. For the innovators pursuing exploitative innovation, the attachment strategy with negative preference is a better choice. However, when the network characteristics are particularly sensitive to the innovation cost and do not require high innovation speed, a strong positive preference strategy is a better choice.

The research of this paper can also be extended in the following aspects. First, although this paper explores the influence of different attachment preferences on the structural characteristics of the innovative network, it is limited to the discussion of the overall structural form of the network. On this basis, future studies can conduct in-depth analysis of myriad indices of network characteristics, such as those of network centralities, differences in node status, and aggregation characteristics, under different attachment preferences. Second, the computer simulation research method is adopted in this paper. The conclusions need to be further verified by empirical research. Third, the innovation model in this paper mainly describes the generation phase of innovation. However, there will be completely different knowledge dynamics after innovation occurs. Therefore, the conclusions of this paper do not apply to subsequent phases, such as the acceptance and implementation of innovations. An effective attachment strategy and optimal network structure in subsequent phases still need to be further explored. These limitations provide opportunities for further study but do not affect the conclusions of this paper.

\section{Data Availability}

The data used to support the findings of this study are generated during the study.

\section{Conflicts of Interest}

The authors declare that there are no conflicts of interest regarding the publication of this paper.

\section{Acknowledgments}

This research was supported by the National Natural Science Foundation of China (Grant no. 71732002), the Humanities and Social Science Fund of Ministry of Education of China (Grant no. 15YJC630034), and the China Postdoctoral Science Foundation (Grant no. 2020M671446).

\section{References}

[1] S. P. Borgatti and D. S. Halgin, "On network theory," Organization Science, vol. 22, no. 5, pp. 1168-1181, 2011.

[2] S. V. Rehm and L. Goel, "Using information systems to achieve complementarity in SME innovation networks," Information \& Management, vol. 54, no. 4, pp. 438-451, 2016.

[3] A. L. J. Ter Wal, P. Criscuolo, B. McEvily, and A. Salter, "Dual networking: how collaborators network in their quest for innovation," Administrative Science Quarterly, vol. 65, no. 4, pp. 887-930, 2020.

[4] M. Mitrega, S. Forkmann, G. Zaefarian, and S. C. Henneberg, "Networking capability in supplier relationships and its impact on product innovation and firm performance," International Journal of Operations \& Production Management, vol. 37, no. 5, pp. 577-606, 2017.

[5] E. Fang, "The effect of strategic alliance knowledge complementarity on new product innovativeness in China," Organization Science, vol. 22, no. 1, pp. 158-172, 2011.

[6] C. Iturrioz, C. Aragón, and L. Narvaiza, "How to foster shared innovation within SMEs' networks: social capital and the role of intermediaries," European Management Journal, vol. 33, no. 2, pp. 104-115, 2015.

[7] M. E. Newman, "Clustering and preferential attachment in growing networks," Physical review. E, Statistical, nonlinear, and soft matter physics, vol. 64, no. 2, Article ID 025102, 2001.

[8] H. Jeong, Z. Néda, and A. L. Barabási, "Measuring preferential attachment in evolving networks," Europhysics Letters, vol. 61, no. 4, pp. 567-572, 2003.

[9] A. Capocci, V. D. Servedio, F. Colaiori et al., "Preferential attachment in the growth of social networks: the internet encyclopedia Wikipedia," Physical review. E, Statistical, nonlinear, and soft matter physics, vol. 74, no. 3, Article ID 036116, 2006.

[10] E. Eisenberg and E. Y. Levanon, "Preferential attachment in the protein network evolution," Physical Review Letters, vol. 91, no. 13, Article ID 138701, 2003.

[11] D. Wang, C. Song, and A.-L. Barabási, "Quantifying longterm scientific impact," Science, vol. 342, no. 6154, pp. 127-132, 2013.

[12] A. M. Petersen, W.-S. Jung, J.-S. Yang, and H. E. Stanley, "Quantitative and empirical demonstration of the Matthew effect in a study of career longevity," Proceedings of the $\mathrm{Na}$ tional Academy of Sciences, vol. 108, no. 1, pp. 18-23, 2011. 
[13] R. D. Raizada and M. M. Kishiyama, "Effects of socioeconomic status on brain development, and how cognitive neuroscience may contribute to levelling the playing field," Frontiers in Human Neuroscience, vol. 4, p. 3, 2010.

[14] A.-L. Barabási and R. Albert, "Emergence of scaling in random networks," Science, vol. 286, no. 5439, pp. 509-512, 1999.

[15] A. L. Barabási, R. Albert, and H. Jeong, "Mean-field theory for scale-free random networks," Physica A: Statistical Mechanics and Its Applications, vol. 272, no. 1-2, pp. 173-187, 1999.

[16] M. Golosovsky and S. Solomon, "The transition towards immortality: non-linear autocatalytic growth of citations to scientific papers," Journal of Statistical Physics, vol. 151, no. 12, pp. 340-354, 2013.

[17] A. L. Barabási, H. Jeong, Z. Néda, E. Ravasza, A. Schubertd, and T. Vicsekbe, "Evolution of the social network of scientific collaborations," Physica A: Statistical Mechanics and Its Applications, vol. 311, no. 3-4, pp. 590-614, 2002.

[18] M. Tomassini and L. Luthi, "Empirical analysis of the evolution of a scientific collaboration network," Physica A: Statistical Mechanics and Its Applications, vol. 385, no. 2, pp. 750-764, 2007.

[19] B. F. de Blasio, A. Svensson, and F. Liljeros, "Preferential attachment in sexual networks," Proceedings of the National Academy of Sciences, vol. 104, no. 26, pp. 10762-10767, 2007.

[20] M. Golosovsky and S. Solomon, "Stochastic dynamical model of a growing citation network based on a self-exciting point process," Physical Review Letters, vol. 109, no. 9, Article ID 098701, 2012.

[21] D. M. Pennock, G. W. Flake, S. Lawrence, E. J. Glover, and C. L. Giles, "Winners don't take all: characterizing the competition for links on the web," Proceedings of the National Academy of Sciences, vol. 99, no. 8, pp. 5207-5211, 2002.

[22] P. L. Krapivsky, S. Redner, and F. Leyvraz, "Connectivity of growing random networks," Physical Review Letters, vol. 85, no. 21, pp. 4629-4632, 2000.

[23] S. N. Dorogovtsev, J. F. F. Mendes, and A. N. Samukhin, "Structure of growing networks with preferential linking," Physical Review Letters, vol. 85, no. 21, pp. 4633-4636, 2000.

[24] R. Reagans and B. McEvily, "Network structure and knowledge transfer: the effects of cohesion and range," Administrative Science Quarterly, vol. 48, no. 2, pp. 240-267, 2003.

[25] B. Shaw-Ching Liu, R. Madhavan, and D. Sudharshan, "DiffuNET: the impact of network structure on diffusion of innovation," European Journal of Innovation Management, vol. 8, no. 2, pp. 240-262, 2005.

[26] P. Rohani, X. Zhong, and A. A. King, "Contact network structure explains the changing epidemiology of pertussis," Science, vol. 330, no. 6006, pp. 982-985, 2010.

[27] R. Hausmann and C. A. Hidalgo, "The network structure of economic output," Journal of Economic Growth, vol. 16, no. 4, pp. 309-342, 2011.

[28] Y. H. Eom and S. Fortunato, "Characterizing and modeling citation dynamics," PLoS One, vol. 6, no. 9, Article ID e24926, 2011.

[29] M. S. Granovetter, "The strength of weak ties," American Journal of Sociology, vol. 78, no. 6, pp. 1360-1380, 1973.

[30] M. T. Hansen, "The search-transfer problem: the role of weak ties in sharing knowledge across organization subunits," Administrative Science Quarterly, vol. 44, no. 1, pp. 82-111, 1999.

[31] R. S. Burt, "Structural holes and good ideas," American Journal of Sociology, vol. 110, no. 2, pp. 349-399, 2004.
[32] G. Ahuja, "Collaboration networks, structural holes, and innovation: a longitudinal study," Administrative Science Quarterly, vol. 45, no. 3, pp. 425-455, 2000.

[33] R. Cowan and N. Jonard, "Network structure and the diffusion of knowledge," Journal of Economic Dynamics and Control, vol. 28, no. 8, pp. 1557-1575, 2004.

[34] L. Hua and W. Wang, "The impact of network structure on innovation efficiency: an agent-based study in the context of innovation networks," Complexity, vol. 21, no. 2, pp. 111-122, 2015.

[35] J. Björk and M. Magnusson, "Where do good innovation ideas come from? Exploring the influence of network connectivity on innovation idea quality," Journal of Product Innovation Management, vol. 26, no. 6, pp. 662-670, 2009.

[36] U. Andersson, À. Dasí, R. Mudambi, and T. Pedersen, "Technology, innovation and knowledge: the importance of ideas and international connectivity," Journal of World Business, vol. 51, no. 1, pp. 153-162, 2016.

[37] V. Gilsing, B. Nooteboom, W. Vanhaverbeke, G. Duysters, and A. van den Oord, "Network embeddedness and the exploration of novel technologies: technological distance, betweenness centrality and density," Research Policy, vol. 37, no. 10 , pp. 1717-1731, 2008.

[38] C. C. Phelps, "A longitudinal study of the influence of alliance network structure and composition on firm exploratory innovation," Academy of Management Journal, vol. 53, no. 4, pp. 890-913, 2010.

[39] T. Chen and Z.-G. Shao, "Power-law accelerating growth complex networks with mixed attachment mechanisms," Physica A: Statistical Mechanics and Its Applications, vol. 391, no. 8, pp. 2778-2787, 2012.

[40] U. Wilensky, NetLogo (And NetLogo User Manual), Center for Connected Learning and Computer-Based Modeling, Northwestern University, Evanston, IL, USA, 1999.

[41] L. Fleming, "Recombinant uncertainty in technological search," Management Science, vol. 47, no. 1, pp. 117-132, 2001.

[42] W. S. Lovejoy and A. Sinha, "Efficient structures for innovative social networks," Management Science, vol. 56, no. 7, pp. 1127-1145, 2010.

[43] D. Vera and M. Crossan, "Strategic leadership and organizational learning," Academy of Management Review, vol. 29, no. 2, pp. 222-240, 2004.

[44] J. J. P. Jansen, F. A. J. Van Den Bosch, and H. W. Volberda, "Exploratory innovation, exploitative innovation, and performance: effects of organizational antecedents and environmental moderators," Management Science, vol. 52, no. 11, pp. 1661-1674, 2006.

[45] R. Duane Ireland and J. W. Webb, "Strategic entrepreneurship: creating competitive advantage through streams of innovation," Business Horizons, vol. 50, no. 1, pp. 49-59, 2007.

[46] J. G. March, "Exploration and exploitation in organizational learning," Organization Science, vol. 2, no. 1, pp. 71-87, 1991.

[47] Z.-L. He and P.-K. Wong, "Exploration vs. exploitation: an empirical test of the ambidexterity hypothesis," Organization Science, vol. 15, no. 4, pp. 481-494, 2004.

[48] D. R. Gibson, "Concurrency and commitment: network scheduling and its consequences for diffusion1," Journal of Mathematical Sociology, vol. 29, no. 4, pp. 295-323, 2005. 